\title{
Molecular dynamics simulations of the delta and omicron SARS-CoV-2 spike - ACE2 complexes reveal distinct changes between both variants
}

\section{Eileen Socher}

Friedrich-Alexander-University Erlangen-Nürnberg

\section{Lukas Heger}

University Hospital Erlangen

\section{Friedrich Paulsen}

Friedrich-Alexander-University Erlangen-Nürnberg

\section{Friederike Zunke}

University Hospital Erlangen

Philipp Arnold ( $\sim$ Philipp.Arnold@fau.de)

Friedrich-Alexander-University Erlangen-Nürnberg

\section{Article}

Keywords: COVID-19, SARS-CoV-2, B.1.427/B.1.429, B.1.617.2, B.1.617.1, B.1.1.529, epsilon variant, delta variant, kappa variant, omicron variant, VOC, spike protein, receptor-binding domain, ACE2, receptor interface, molecular dynamics simulations

Posted Date: January 12th, 2022

DOI: https://doi.org/10.21203/rs.3.rs-1228784/v1

License: @ (i) This work is licensed under a Creative Commons Attribution 4.0 International License. Read Full License

Version of Record: A version of this preprint was published at Computational and Structural Biotechnology Journal on February 1st, 2022. See the published version at https://doi.org/10.1016/j.csbj.2022.02.015. 


\section{Molecular dynamics simulations of the delta and omicron SARS-CoV-2 spike - ACE2 complexes reveal distinct changes between both variants}

\section{Eileen Socher ${ }^{1,2}$, Lukas Heger ${ }^{3}$, Friedrich Paulsen ${ }^{1,4}$, Friederike Zunke ${ }^{5}$ and} Philipp Arnold ${ }^{1}$

${ }^{1}$ Institute of Anatomy, Functional and Clinical Anatomy, Friedrich-Alexander University Erlangen-Nürnberg (FAU), Erlangen, Germany; eileen.socher@fau.de (E.S.); friedrich.paulsen@fau.de (F.P.); philipp.arnold@fau.de (P.A.)

${ }^{2}$ Institute for Clinical and Molecular Virology, Friedrich-Alexander University Erlangen-Nürnberg (FAU), University Hospital Erlangen, Erlangen, Germany; eileen.socher@fau.de

${ }^{3}$ Laboratory of Dendritic Cell Biology, Department of Dermatology, Friedrich-Alexander University Erlangen-Nürnberg (FAU), University Hospital Erlangen, Erlangen, Germany; lukas.heger@uk-erlangen.de

${ }^{4}$ Sechenov University, Department of Operative Surgery and Topographic Anatomy, 119992 Moscow, Russia; friedrich.paulsen@fau.de

${ }^{5}$ Department of Molecular Neurology, University Hospital Erlangen, Friedrich-Alexander University Erlangen-Nürnberg (FAU), Erlangen, Germany; friederike.zunke@fau.de

*Correspondence: eileen.socher@fau.de (E.S.) and philipp.arnold@fau.de (P.A.);

Keywords: COVID-19, SARS-CoV-2, B.1.427/B.1.429, B.1.617.2, B.1.617.1, B.1.1.529, epsilon variant, delta variant, kappa variant, omicron variant, VOC, spike protein, receptor-binding domain, ACE2, receptor interface, molecular dynamics simulations 
SARS-CoV-2, the virus which causes the COVID-19 pandemic, changes frequently through the appearance of mutations constantly leading to new variants. However, only few variants evolve as dominating and will be considered as "Variants of Concern" (VOCs) by the world health organization (WHO). At the end of 2020 the alpha (B.1.1.7) variant appeared in the United Kingdom and dominated the pandemic situation until mid of 2021 when it was substituted by the delta variant (B.1.617.2) that first appeared in India as predominant variant. At the end of 2021, SARS-CoV-2 omicron (B.1.1.529) evolved as the dominating variant. Here, we use in silico modeling and molecular dynamics (MD) simulations of the receptor-binding domain of the viral spike protein and the host cell surface receptor ACE2 to analyze and compare the interaction pattern between the wild type, delta and omicron variants. We identified residue 493 in delta (glutamine) and omicron (arginine) with altered binding properties towards ACE2.

\section{Introduction}

The COVID-19 pandemic caused by the novel severe acute respiratory syndrome coronavirus 2 (SARSCoV-2) is driven by newly emerging variants that arise from the original virus. Although most of the genetic changes have little to no impact on the virus' properties, some virus variants arise with higher transmissibility and/or increased virulence. These variants are classified as "Variants of Concern" (VOC) by the WHO. In January 2022, the WHO listed five VOCs labeled with Greek letters ${ }^{1}$ : (1) the alpha variant (Pango lineage: B.1.1.7) that emerged in the United Kingdom, (2) the beta variant (Pango lineage: B.1.351) from South Africa, (3) the gamma variant (Pango lineage: P.1) from Brazil, (4) the delta variant (Pango lineage: B.1.617.2) first detected in India and the omicron variant (Pango lineage: B.1.1.529) first identified in South Africa in November $2021^{2}$.

All these VOCs listed by the WHO acquired some distinctive mutations (Fig. 1a lists the mutations of the predominant delta and omicron variants) in the trimeric spike glycoprotein of SARS-CoV-2, which specifically binds with its receptor-binding domain (RBD) to the host cell receptor angiotensin-converting enzyme $2(A C E 2)^{3-5}$. This binding step is crucial for viral entry into a host cell to initiate infection (Fig. 1b) ( $^{6,7}$. Therefore, understanding the interaction of the virus with ACE2 at the cell surface is fundamentally important in the fight against SARS-CoV-2. The protein structure of the RBD of the wild type spike protein in complex with ACE2 is known (e.g. PDB ID code: $7 \mathrm{KMB}^{8}$ ) and allows characterization of receptor binding at the atomic level, greatly improving our understanding of host-pathogen interactions at this site. By introducing the observed mutations into the wild type RBD-ACE2 complex structure with in silico modeling and subsequently conducting molecular dynamics (MD) simulations of these RBD-ACE2 complexes, the influence of specific mutations in the RBD on binding towards the human receptor can be strongly enhanced ${ }^{9-11}$. Experimentally determined protein structures provide the basis for MD simulations, which can add information on the protein dynamics, the flexibility and residue interactions that would be difficult to access with experimental methods. Additionally, MD simulations can today often be performed and analyzed much faster than experimentally determined structures can be obtained and therefore it can serve as a predictive tool.

The mentioned alpha, beta, gamma and omicron variants harbor as common feature all a substitution of asparagine-to-tyrosine at position 501 (N501Y) in the RBD, which is not present in the delta variant ${ }^{12}$. In addition to the N510Y mutation, omicron carries 14 other mutations within the RBD (Fig. 1a). A considerable number of them can be found at the direct binding interface with ACE2 (Fig. 1b). In contrast to omicron, the delta variant has a characteristic leucine-to-arginine substitution at position 452 (L452R), which was also earlier detected in the related kappa variant (B.1.617.1) and in an unrelated lineage in the United States of America (epsilon variant: B.1.427/B.1.429). For variants carrying this L452R mutation, stronger affinity of the spike protein for the ACE2 receptor was described ${ }^{13,14}$. The 
delta and omicron variants share a common T478K mutation and for delta reduced affinity of neutralizing antibodies at this position was shown ${ }^{15-17}$. In omicron many of the mutations found at the RBD are located within epitopes recognized by neutralizing antibodies. An exemplary array of such neutralizing antibodies is shown in Supplementary Fig. 1 and experimental data suggests reduced binding of antibodies from patient sera ${ }^{18,19}$. Of note, especially antibodies binding at the RBD at the site of contact formation with ACE2 show high neutralization properties ${ }^{20}$.

The present study compares the original wild type (wt), the delta variant and the similar variants epsilon and kappa with the omicron variant. We identify residue 493 within the RBD as a major difference between all three variants. In wt and delta residue 493 is a glutamine (Gln493) and we show that the delta variant amino acid exchange $L 452 \mathrm{R}$ induces a rearrangement in all three L452R carrying variants (epsilon, delta and kappa) that increase the number of contacts and the number of hydrogen bonds with ACE2 for Gln493. The omicron variant expresses an arginine at position 493 and this arginine forms very stable salt bridges with residues glutamate 35 (Glu35) and aspartate 38 (Asp38) of the host cell receptor ACE2. In previous reports ${ }^{21}$ an exchange from glutamine to lysine was reported at position 493 and we can show, that the omicron variants with Q493R or Q493K show very similar changes in contact formation with ACE2. However, for Q493R we also identified salt bridge formation with aspartate 30 (Asp30) that was not observed for the Q493K mutation. Additionally, omicron also carries an amino acid exchange from lysine 417 to asparagine (K417N). The same mutation was already observed in the beta variant, while in the gamma variant lysine 417 is mutated to a threonine (K417T). In wild type, Lysine 417 forms a very stable salt bridge with aspartate 30 from the ACE2 receptor and we could show that exchange to asparagine or threonine largely disrupts binding at this position? ${ }^{9}$.

Here, we show that major changes in interaction with ACE2 occur at positions 417, 493, 501 and at position 505 where a tyrosine is mutated to a histidine ( $\mathrm{Y} 505 \mathrm{H})$. We also show, that many of the remaining amino acid exchanges occurring at the interface to ACE2 do not largely influence binding to ACE2, but rather change epitopes for neutralizing antibodies ${ }^{20}$, which might explain the reduced neutralization capacity identified for serum samples from vaccinated patients ${ }^{18}$.

\section{Results}

\section{The contact analyses and electrostatic potential of the viral receptor-binding domain}

The SARS-CoV-2 delta and omicron variants have both acquired a threonine-to-leucine (T478K) substitution in the RBD. The delta variant carries an additional leucine-to-arginine (L452R) substitution in the RBD and the omicron variant has, in addition to this T478K mutation, 14 other mutations within the RBD (Fig. 1a). In order to compare individual virus variants, we performed four independent MD simulation runs over $500 \mathrm{~ns}$ for every variant's RBD in complex with ACE2. Subsequently, we analyzed the overall intermolecular contacts formed between the human ACE2 and the viral RBD of the wild type and the delta and omicron variants. We found a reduced number of contacts between the RBD and ACE2 for the omicron variant, while the number of contacts for the delta variant was not markedly changed (Fig. 1C). Then, we assigned the individual number of contacts to all RBD amino acids within $8 \AA$ A distance to ACE2 and found marked differences for residues 493, 501 and 505 (Fig. 1d, Supplementary Fig. 1a). Wild type and delta variant express an asparagine at position 501, whereas the omicron variant harbors a tyrosine (N501Y) at this position. The functional consequences of this N501Y mutation were already described ${ }^{22}$ and a cryo-electron microscopy structure carrying this N501Y exchange in complex with ACE2 was also experimentally solved (PDB ID code: $7 \mathrm{MJN}^{23}$ ). In the omicron variant, the tyrosine at position 505 (wt and delta variant) is mutated to histidine and we identified a reduced number of contacts to ACE2 for histidine at this position (Fig. 1d, Supplementary Fig. 2a and Supplementary Fig. 2b). In detail, residue 505 interacts with the carbon atoms of the side chain of lysine 353 on ACE2 (Supplementary Fig. 2c). Here a pi-electron ring system (as for tyrosine expressing variants) 
might have more contacts than the inserted histidine in omicron. For the epsilon (B.1.427/B.1.429) and kappa (B.1.617.1) variant, we also performed contact analyses and found no marked differences on the individual amino acid level (Supplementary Fig. $2 \mathrm{~d}$ ). As the omicron variant shows a considerable number of positively charged amino acids at sites of mutation, we analyzed the electrostatic potential at the RBD-ACE2 interface and found increased electro positivity in the delta variant (L452R and T478K) and even more pronounced in the omicron variant (T478K, Q493R, Q498R and Y505H; Fig. 1e). However, the omicron variant also loses a positively charged amino acid at the interaction interface at position 417 (K417N; Fig. 1b, Fig. 1e).

124
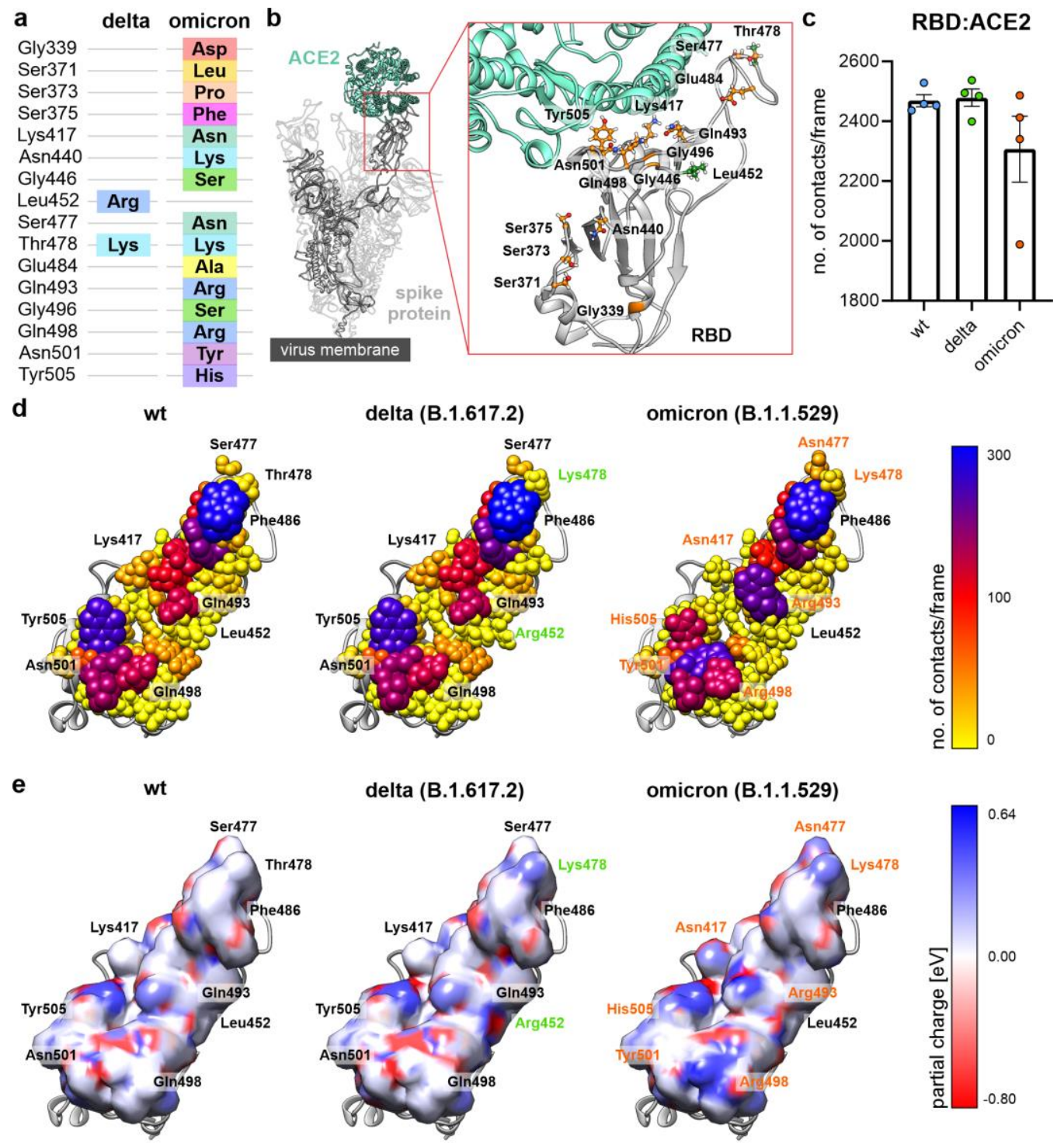

Figure 1: Number of contacts and electrostatic potential. a, Amino acid exchanges at distinct positions in the delta and omicron variants. $\mathbf{b}$, Structural representation of the SARS-CoV-2 spike protein on the viral membrane with the inset showing all residues mutated in delta (B.1.617.2, green) or omicron (B.1.1.529, orange). c, Number of contacts between the RBD and ACE2 for wt, delta and omicron. $d$, Structural representation of the interface of the RBD with ACE2 according to the number of contacts in color code. Residues with a green label are mutated in the delta variant and residues with an orange label are mutated in omicron. e, Surface representation of the interface formed by the RBD with ACE2. Surface coloring according to the electrostatic potential of the underlying residues with $0.64 \mathrm{eV}$ in blue and $-0.80 \mathrm{eV}$ in red. 
135 As numerous positively charged amino acids appear at the interaction interface, we also analyzed the linear electrostatic interaction energy to identify newly formed or resolved intermolecular salt bridges. Largest differences between wt, delta and omicron were found at positions 417 and 493 (Fig. 2a, Supplementary Fig. 3). Analyzing the electrostatic potential at the interaction interface on ACE2 revealed aspartate 30 , glutamate 35 and aspartate 38 as potential interaction partners for these two residues. For other variants that exchanged lysine 417 to asparagine or threonine, we already identified a strongly reduced electrostatic interaction energy with aspartate $30^{9}$. Thus it was not very surprising that we found the same reduction for the omicron variant (Fig. 2c) and a salt bridge in wt (Fig. 2d) that also remains stable over time (Fig. 2e). Next, we analyzed contact formation for residue 493 (Fig. 2f). We found that wt and delta (both with Gln493) behave similar, but that omicron shows some major differences. The delta variant shows a small increase in contact formation with lysine 31 and glutamate 35(Fig. 2f). In contrast, omicron changes its interaction profile completely. For one MD simulation run we identified interaction with aspartate 30 (Asp30) and in three runs increased interaction with glutamate 35 (Glu35) and with aspartate 38 (Asp38). Additionally, the interaction with lysine 31 (Lys31) is reduced in all four simulation runs of the omicron variant (Fig. 2f) probably due to electrostatic repulsion between the positively charged arginine 493 and the also positively charged lysine 31 . 
a

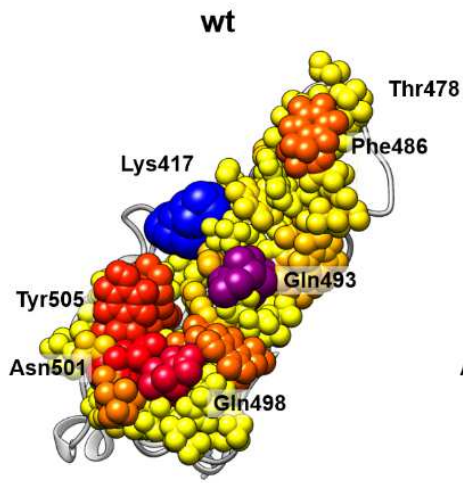

b

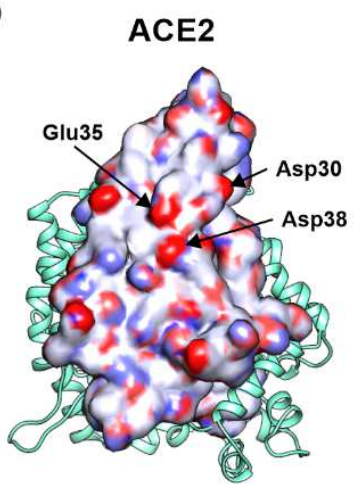

delta (B.1.617.2)

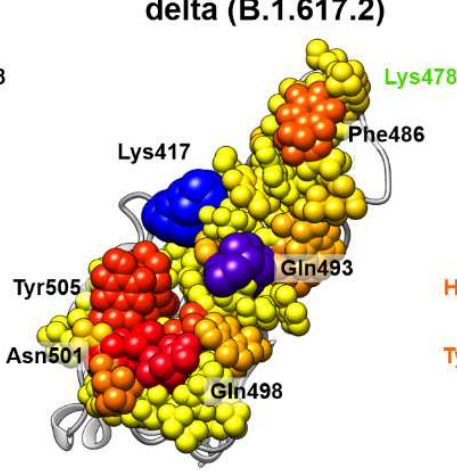

C

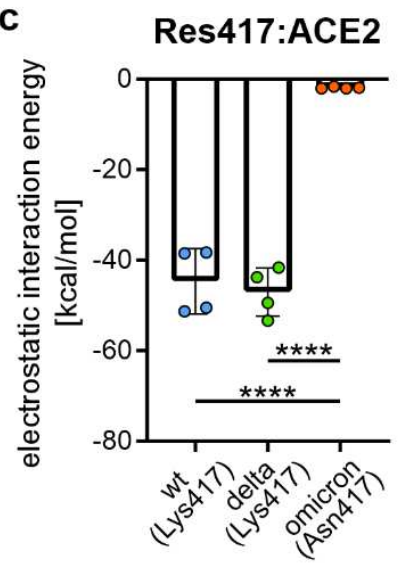

omicron (B.1.1.529)

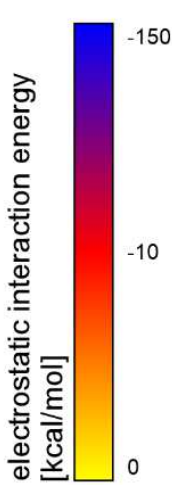

d

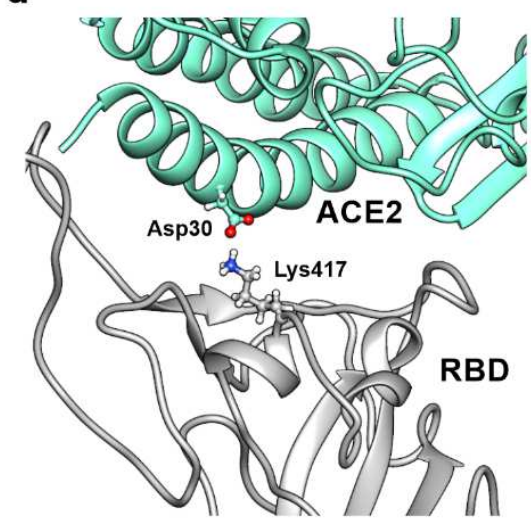

e
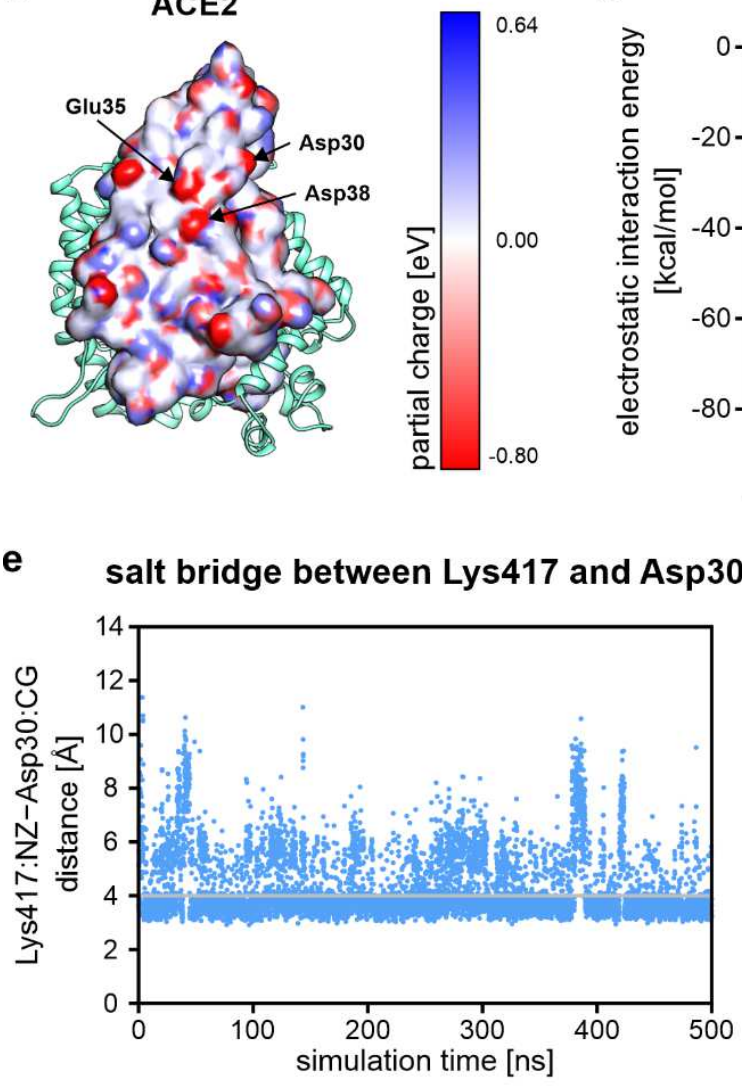

Figure 2: Linear electrostatic interaction shows two major changes in omicron. a, Structural representation of the RBD interface with ACE2 with residues shown as spheres with their size and color according to their electrostatic interaction. $\mathbf{b}$, Surface representation of the binding interface of ACE2 with the RBD. Surface color is labeled according to the partial charge of the underlying residues with the three negatively charged residues aspartate 30 (Asp30), glutamate 35 (Glu35) and aspartate 38 (Asp38). c, Linear electrostatic binding energy of residue 417 in wt (blue), delta (green) and omicron (orange; ${ }^{* * * *} p<0.0001$ one-way ANOVA). d, Structural representation of the salt bridge formed by lysine 417 (Lys417) from the RBD and aspartate 30 (Asp30) from ACE2 in the wt complex. e, Time resolved distance plot of the distance between side chain atoms of Lys417 and Asp30 in the wt complex. f, Number of contacts per frame formed by residue 493 with interacting ACE2 residues.

\section{The delta variant shows increased hydrogen bond formation with ACE2}

To analyze changes in the delta variant, we pooled it with four individual MD simulation runs of the epsilon and four runs of the kappa variant (all these variants carry the L452R mutation) and compared 
it to twelve 500 ns long simulation runs of the wt RBD-ACE2 complex (eight of them were already described earlier $\left.{ }^{9,10}\right)$. Within the neighboring beta sheet of glutamine 493 , the L452R exchange can be found (Fig. 3a, b), thereby changing the structural environment of glutamine 493 . We found that the number of intermolecular contacts between glutamine 493 and the human receptor ACE2 increases in the different L452R variants compared to wild type RBD-ACE2 complexes (Fig. 3C). Decomposed on the individual amino acid level, although not significant, we identified the ACE2 residues glutamate 35 and to a minor extend lysine 31 with an increased number of contacts to glutamine 493 (Fig. 3d,e). Contact formation between glutamine 493 and histidine 34 of ACE2 was unchanged, however (Fig. $3 \mathrm{~d}, \mathrm{e})$. As glutamine residues engage in hydrogen bonds, we also analyzed the number of intermolecular hydrogen bonds of glutamine 493 with ACE2 and found a significant increase for variants that express the L452R mutation (Fig. 3f). Detailed analysis showed an increased occurrence of hydrogen bonds with lysine 31 and with glutamate 35 (Fig. 3g). The structural representation illustrates that the side chain oxygen atom of glutamine 493 forms a hydrogen bond with the side chain of lysine 31 from ACE2 while one of the hydrogens of the amino group forms hydrogen bonds with the oxygen atoms of the carboxyl group of glutamate 35 (Fig. 3h). A detailed root-mean-square fluctuation (RMSF) analysis (Supplementary Fig. 4a) showed a reduced flexibility for the entire glutamine 493 (Supplementary Fig. 4b) and especially for side chain atoms of the amino group (NE2, HE21 and HE22; Supplementary Fig. $4 b$ ) in the different L452R variants compared to wild type RBD-ACE2 complexes. This reduced flexibility of glutamine's side chain atoms can be observed due to the stronger hydrogen bond network of glutamine 493 with ACE2, which stabilizes the side chain position. Histidine 34 does not form hydrogen bonds with glutamine 493, but forms a hydrogen bond with tyrosine 453 (Supplementary Fig. 4c,d). Together, we can conclude that the delta variant shows an increase in contacts and consequently in hydrogen bond formation of glutamine 493 with ACE2 and that this might contribute to an increased binding. 
a

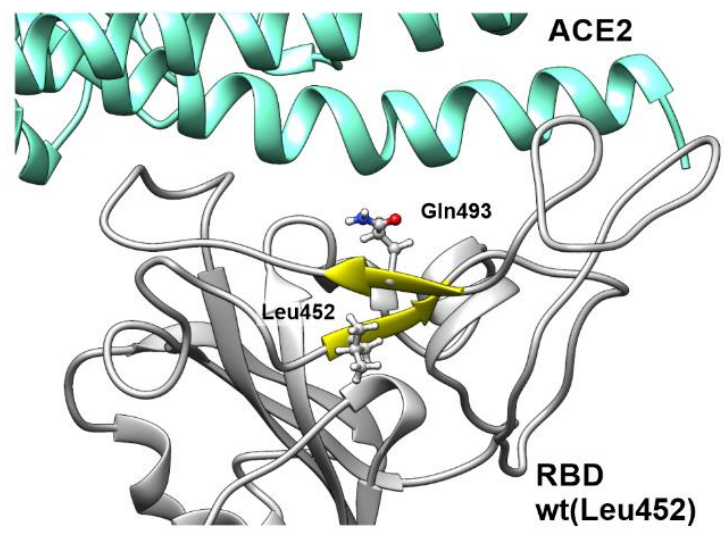

b

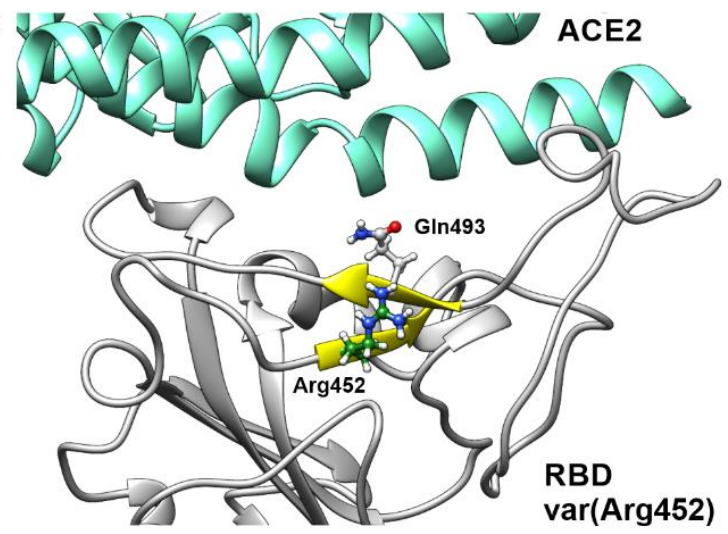

C

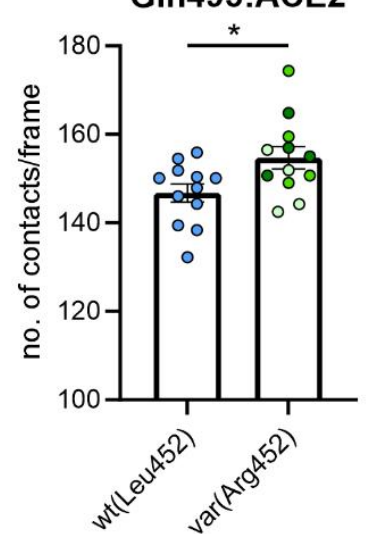

\section{d}

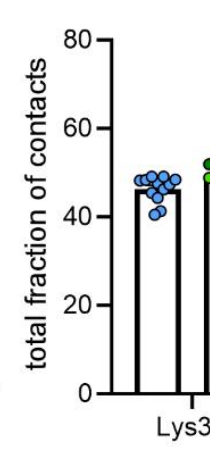

contacts of GIn493

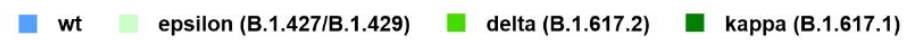

f

GIn493:ACE2 $\mathrm{g}$

hydrogen bonds of GIn493

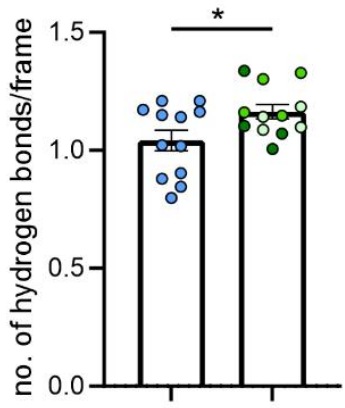

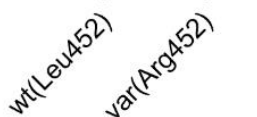

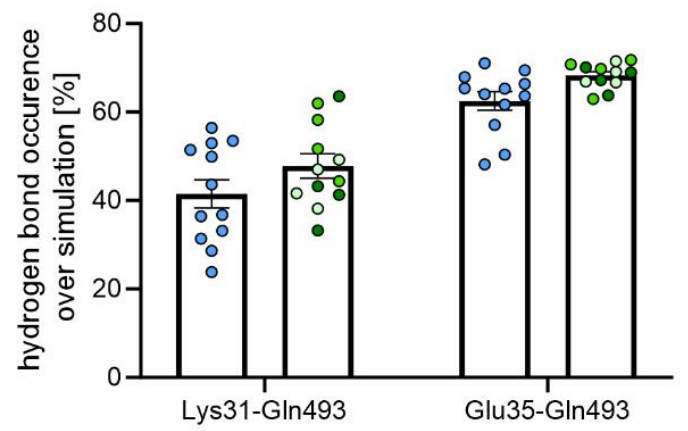

Lys31-GIn493 e

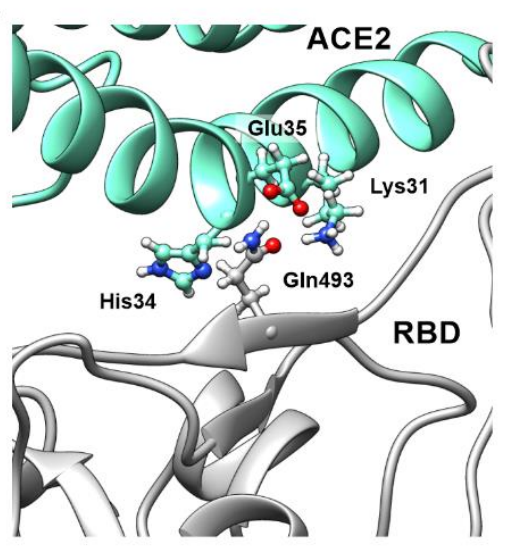

Figure 3: Hydrogen bond rearrangement in delta. a, Structural representation of the glutamine 493 (Gln493) and leucine 452 (Leu452) as expressed on two neighboring $\beta$-strands forming a small 2 -stranded antiparallel $\beta$ sheet (yellow) in wt. b, As in figure panel a, but with residue 452 exchanged to arginine (Arg452, green) as expressed on delta (medium green), epsilon (light green) and kappa (dark green) variants. c, Combined analysis of wt and three variants that carry the leucine 452 arginine (L452R) exchange with regard to the number of contacts formed by glutamine 493 (Gln493) with ACE2 ( ${ }^{*} p<0.05$, two-tailed Student's T-test). d, Residue specific analysis of the number of contacts between wt and L452R expressing variants (one-way ANOVA). e, Structural representation of glutamine 493 (Gln493) expressed on the RBD and its interaction partners from ACE2 lysine 31 (Lys31), histidine 34 (His34) and glutamate 35 (Glu35). f, Number of hydrogen bonds formed by glutamine 493 (Gln493) per frame comparing wt and L452R expressing variants ( ${ }^{*} p<0.05$, two-tailed Student's T-test).g, Hydrogen bond formation between glutamine 493 (Gln493) from the RBD and lysine 31 (Lys31) and glutamate 35 (Glu35, both ACE2). $\mathbf{h}$, Structural representation of the hydrogen bonds formed between glutamine 493 (Gln493, RBD) and lysine 31 (Lys31, ACE2) and glutamate 35 (Glu35, ACE2). 
As described above (Fig. 2f), we identified an entirely altered interaction pattern for arginine at position 493. The number of contacts with glutamate 35 increased and contacts with aspartate 38 were newly formed and not present in residues expressing a glutamine at position 493 (Fig. 2f). For three of our MD simulations, these two newly formed salt bridges can also be appreciated in time course distance plots of arginine 493 with aspartate 30, glutamate 35 and aspartate 38 (Fig. 4a; Supplementary Fig. 5a). However, one of our MD simulation runs showed a different picture and we found a stable intermolecular salt bridge between arginine 493 and aspartate 30 on ACE2 (Fig. 4b). Analyzing the average distances to favored residues on ACE2 within single runs provided insight into the stability of the individual salt bridges (Fig. 4c). Especially interactions between arginine 493 and aspartate 30 and glutamate 35 show low standard deviations with average distances below $5 \AA$, thus strongly supporting ionic interaction. Analysis of the linear electrostatic interaction energy at position 493 showed a strong increase for omicron when compared to wt and delta (Fig. 4d). Thus, we can now postulate two different interactions with ACE2 for arginine at position 493 in the omicron variant. In the first mode, it forms salt bridges with glutamate 35 and aspartate 38 (Fig. 4e) and in the second mode it forms a salt bridge with aspartate 30 (Fig. 4f). Within the same simulation run, where we identified a stable salt bridge between arginine 493 and aspartate 30 from ACE2, we also identified a stable salt bridge between arginine 498 (only present in omicron) and aspartate 38 (Fig. 4f, Supplementary Fig. 5b). Together, omicron carrying an arginine at position 493 (Q493R) shows a higher flexibility in contact formation than wt or delta in the same region. As there was a sequence conflict at position 493 at the beginning, we also simulated the RBD-ACE2 complex with a lysine at this position 493. All runs showed stable salt bridges between lysine 493 and glutamate 35 (Supplementary Fig. 6a,b) and aspartate 38 (Supplementary Fig. $6 c$,d) of ACE2. A lysine side chain at this position might be too short to interact with aspartate 30 (Supplementary Fig. 6e). The increase in linear electrostatic interaction energy, however, remains the similar (Supplementary Fig. 6f). One selection advantage of the omicron over the omicron+Q493K variant might be the more flexible interaction regime at this position. 
$\mathbf{a}$
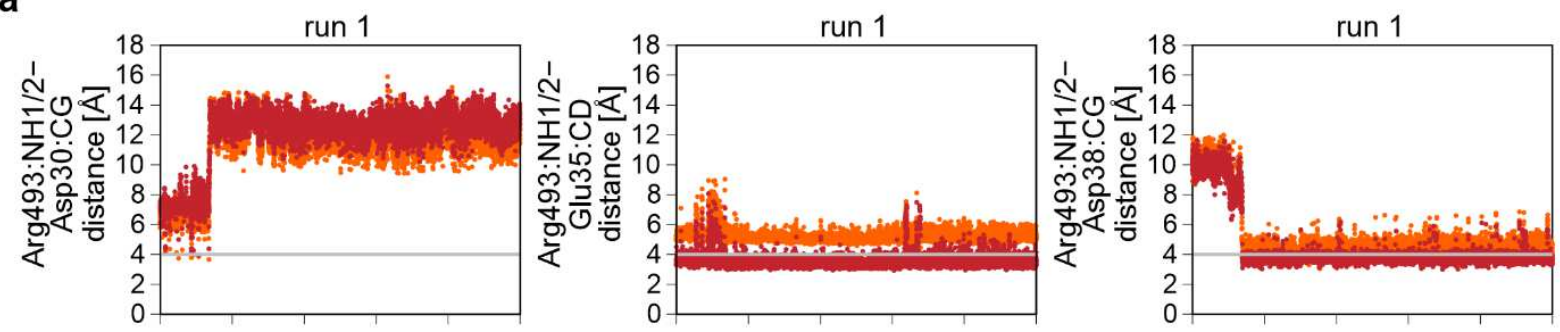

b

run 3

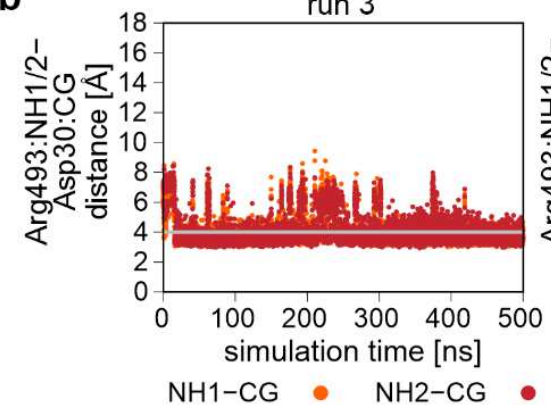

run 3

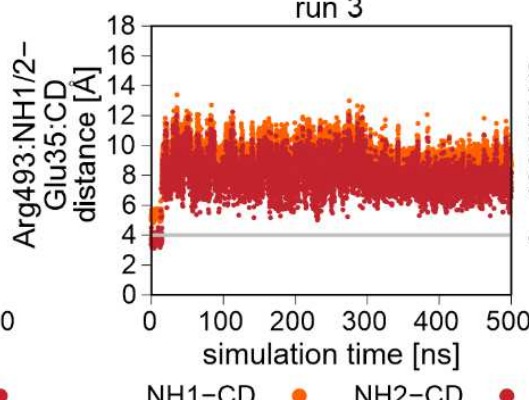

run 3

C

salt bridges of Arg493

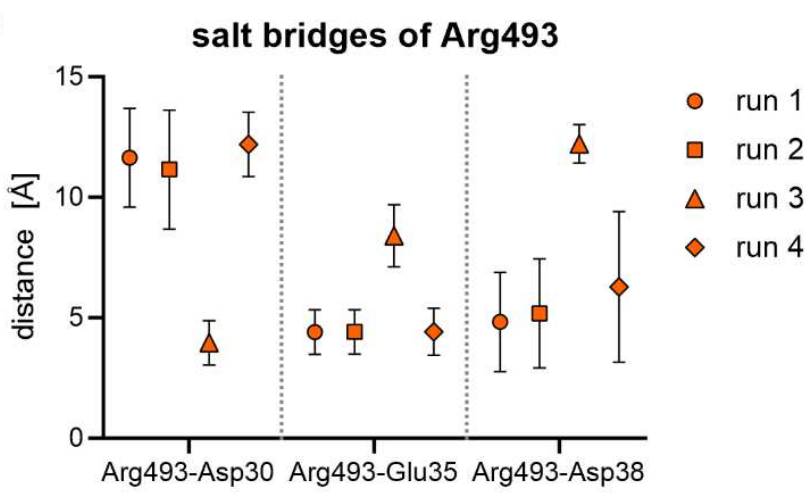

d
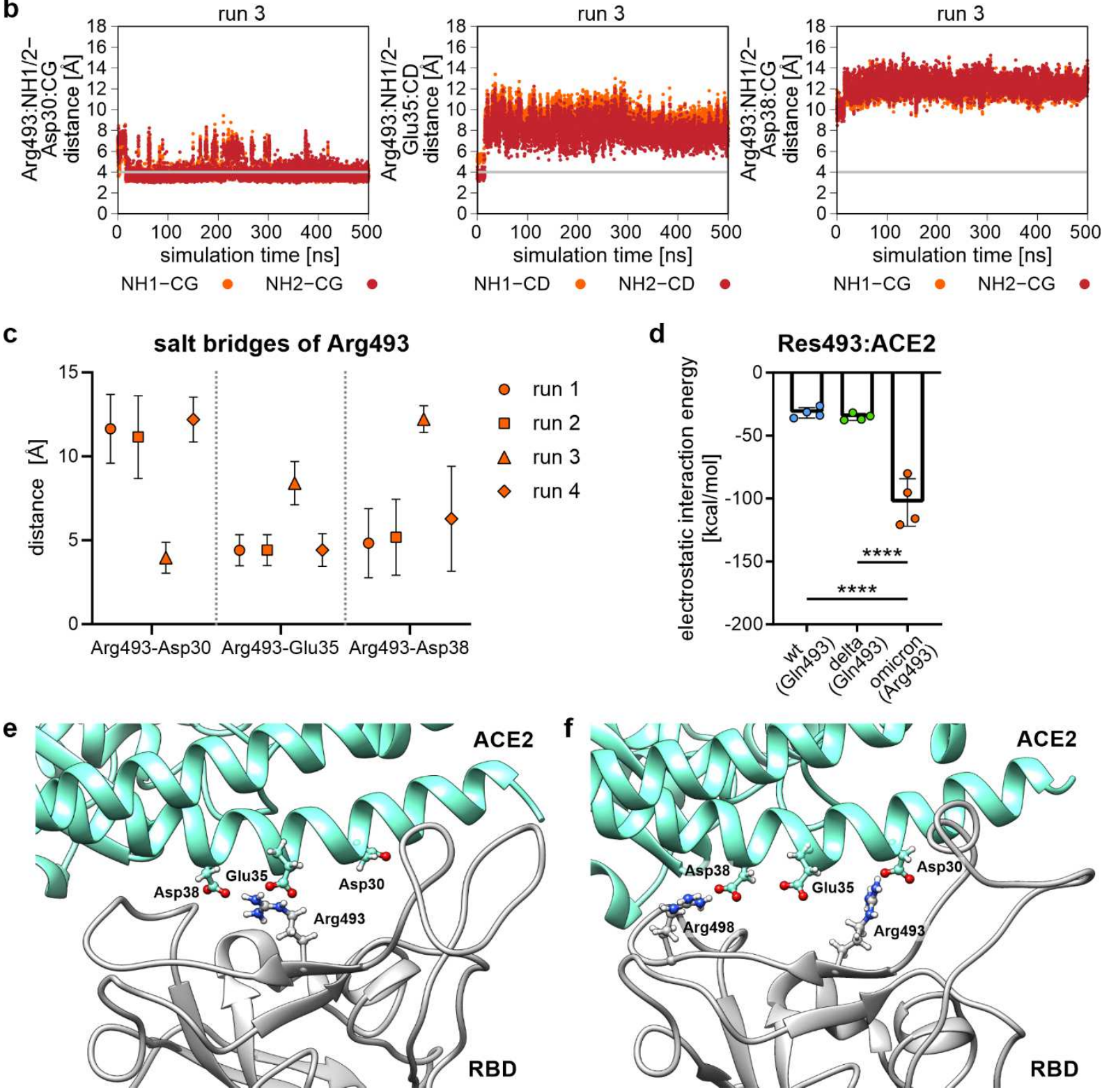

Figure 4: Newly formed salt bridges in omicron between arginine 493 and negatively charged residues on ACE2.

a, Representative distance plots of arginine 493 (RBD, omicron) and aspartate 30 (Asp30), glutamate 35 (Glu35) and aspartate 38 (Asp38) (all three on ACE2). b. Distance plots of a different MD simulation run than shown in figure panel a between the same residues. c, Average distance with standard deviation error bars shown for four individual 500 ns runs for residues arginine 493 (RBD, omicron) and aspartate 30 (Asp30), glutamate 35 (Glu35) and aspartate 38 (Asp38). d, Linear electrostatic interaction energy for residue 493 analyzed for wt (blue), delta (green) and omicron (orange; $* * * * \mathrm{p}<0.0001$, one-way ANOVA). e, Structural representation of the two newly formed salt bridges between arginine 493 (Arg493) on the RBD and glutamate 35 (Glu35) and aspartate 38 (Asp38) both expressed on ACE2. f, Structural representation of salt bridges formed between Arg493 (RBD) and Asp30 (ACE) and Arg498 (RBD) and Asp38 (ACE2). 
The appearance of a L452R exchange within the RBD of the SARS-CoV-2 spike protein rendered the affected variants to former "Variants under Investigation" (epsilon, kappa) or current "Variant of Concern" (delta variant) ${ }^{1}$. The delta variant dominated the pandemic worldwide from mid-2021. All three variants carry additional amino acid exchanges within the other parts of the spike protein, including the D614G variant that we identified as a potential molecular switch to liberate the fusion peptide ${ }^{10}$. Here we show, that the L452R mutation induces an increased number of hydrogen bonds formed by glutamine 493 between the RDB (delta variant) and ACE2. This increase, might induce a stronger interaction between both and support increased viral infectivity. Comparing the omicron variant to wt and delta reveals major differences in RBD-ACE2 interaction. As in the alpha variant, omicron carries a $\mathrm{N} 501 \mathrm{Y}$ mutation and, as the beta variant, carries the K417N mutation. The N501Y mutation apparently increases the binding energy between RBD and ACE2 and the K417N mutation reduces $i^{22}$. Before delta dominated the pandemic starting mid-2021, the alpha variant outcompeted the wt SARS-CoV-2 variant end of 2020/beginning of 2021. Parallel with the alpha variant, the beta and gamma variants appeared both with exchanges for lysine at position 417 that induced a loss of the salt bridge at this position ${ }^{9}$. In omicron this salt bridge is also lost. However, through the exchange of glutamine 493 to an arginine (Q493R), two additional salt bridges are formed (either by arginine 493 itself or by arginine 498 ) and a strong increase in linear electrostatic interaction energy can be observed. The variability in salt bridge formation at positions 493 and 498 might increase the probability for RBD-ACE2 interaction and thereby increase infectivity. As a side note, we could also show that a lysine at this position has a less flexible interaction pattern. Overall, three mutations within the RBD of omicron can be attributed to direct changes in interaction with ACE2 and these are K417N, Q493R and N501Y. With the exchange of tyrosine 505 to histidine, a reduced number of contacts is associated. However, the effect of this exchange cannot be fully judged. When comparing the delta and omicron variant to wt an additional trend becomes evident. Amino acid exchanges within the RBD render it more electropositive. Delta is more positive than wt and omicron more positive than delta. This might enable a better passive adhesion to the negatively charged glycolcalyx and here especially to heparin sulfate, which is a critical factor for SARS-CoV-2 binding ${ }^{24-26}$. Thus, we can identify amino acid exchanges which (i) influence direct interaction with ACE2, (ii) change epitopes of neutralizing antibodies and/or (iii) change the electrostatic surface potential. In omicron, five of the fifteen mutations within the RBD insert positively charged amino acids (N440K, T478K, Q493R, Q498R and Y505H) and only one is lost (K417N). All of these six positions are within epitope regions for neutralizing antibodies ${ }^{20}$. In conclusion, omicron started to dominate the pandemic at the end of 2021 with patient numbers rising steeply in affected areas. MD simulation and structural analysis of the RBD-ACE2 complex shows clear differences of omicron to the delta variant. Omicron has progressed in terms of ACE2 binding, in terms of immune escape and presents a more positively charged interface area.

\section{Methods}

\section{Generation of the starting structures}

To investigate the interface between the RBD of the spike protein and ACE2, the respective wild type start structure was taken from the PDB database (PDB ID code: 7KMB (10.1016/j.chom.2020.11.004)). To also generate the starting structures for the MD simulations of the different SARS-CoV-2 variants, the amino acid substitutions (epsilon spike variant (B.1.427/B.1.429): L452R; delta variant (B.1.617.2): L452R and T478K; kappa variant (B.1.617.1): L452R and E484Q, omicron variant (B.1.1.529): G339D, S371L, S373P, S375F, K417N, N440K, G446S, S477N, T478K, E484A, Q493R, G496S, Q498R, N501Y, $\mathrm{Y} 505 \mathrm{H}$ and for an omicron variant with a lysine at position 493: G339D, S371L, S373P, S375F, K417N, 

Swiss-PdbViewer 4.1.027 (http://www.expasy.org/spdbv/).

For the electrostatic analyses of the protein surface, "PQR" output files were generated by using charge parameters assigned with the APBS-PDB2PQR software suite ${ }^{28}$ (https://server.poissonboltzmann.org/).

\section{Molecular dynamics simulations}

Molecular dynamics simulations were performed exactly as described before ${ }^{9,10}$. By using version 20 of the Amber Molecular Dynamics software package (ambermd.org) ${ }^{29}$, the ff14SB force field ${ }^{30}$ and the Amber Tool LEaP, all systems were electrically neutralized with $\mathrm{Na}^{+}$ions and solvated with TIP3P ${ }^{31}$ water molecules. The receptor-binding domain complexed with ACE2 was solvated in a water box with the shape of a truncated octahedron and a distance of at least $25 \AA$ from the borders to the solute.

Minimization was carried out in three consecutive parts to optimize the geometry of the initial structures. In the first minimization part, all water molecules were minimized, while all other atoms were restrained at the initial positions by using a constant force of $10 \mathrm{kcal} \cdot \mathrm{mol}^{-1} \cdot \AA^{-2}$. During the second part, additional relaxation of the sodium ions and the hydrogen atoms of the protein was allowed, while the remaining protein was restrained with $10 \mathrm{kcal} \cdot \mathrm{mol}^{-1} \cdot \AA^{-2}$. In the third part, the entire protein, ions, and water molecules were minimized without any restraints. All three minimization parts started with 2500 steps using the steepest descent algorithm, followed by 2500 steps of a conjugate gradient minimization. After minimization, the systems were equilibrated in two successive steps. In the first step, the temperature was increased from 10 to $310 \mathrm{~K}$ within $0.1 \mathrm{~ns}$ and the protein was restrained with a constant force of $5 \mathrm{kcal} \cdot \mathrm{mol}^{-1} . \AA^{-2}$. In the second step ( $0.4 \mathrm{~ns}$ length), only the $C_{\alpha}$ atoms of the protein were restrained with a constant force of $5 \mathrm{kcal} \cdot \mathrm{mol}^{-1} \cdot \AA^{-2}$. In both equilibration steps, the time step was $2 \mathrm{fs}$. Minimization and equilibration were carried out on CPUs, while the subsequent production runs were performed using pmemd.CUDA on Nvidia A100 GPUs ${ }^{32-34}$. Subsequent 500 ns long production runs were performed without any restraints and at $310 \mathrm{~K}$ (regulated by a Berendsen thermostat ${ }^{35}$ ). Furthermore, the constant pressure periodic boundary conditions with an average pressure of 1 bar and isotropic position scaling were used. For bonds involving hydrogen, the SHAKE algorithm ${ }^{36}$ was applied in the equilibration and production phases. To accelerate the production phase of the MD simulations, hydrogen mass repartitioning $(\mathrm{HMR})^{37}$ was used in combination with a time step of $4 \mathrm{fs}$. For statistical analyses, four independent 500 ns long MD simulation runs were performed for the epsilon, delta, kappa and omicron spike protein RBD variants in complex with ACE2. For the wild type RBD in complex with ACE216 independent 500 ns long MD simulation runs were used (eight completely new MD simulations and in addition also eight MD simulation runs conducted for two earlier studies ${ }^{9,10}$ were reevaluated).

Trajectory analysis (analysis of root-mean-square fluctuations (RMSF), analysis of contacts (always with distance criterion of $\leq 5 \AA$ between any pair of atoms; total fraction of contacts for residue pairs), measurement of interatomic distances, calculation of electrostatic linear interaction energy and hydrogen bond analyses were performed using the Amber tool cpptraj ${ }^{38}$.

\section{Statistics and display}

Statistical analyses were performed with GraphPad Prism (version 9.2.0 for Windows, GraphPad Software, San Diego, California USA, www.graphpad.com) and statistical tests were applied as indicated below the figure. Plots were created in GraphPad and all structure images were made with UCSF Chimera $1.15^{39}$. 
The authors gratefully acknowledge the compute resources and support provided by the Erlangen Regional Computing Center (RRZE) and by NHR@FAU. Supported by the Interdisciplinary Center for Clinical Research (IZKF) at the University Hospital of the University of Erlangen-Nürnberg to F.Z. (JochenKalden funding program N8) and P.A. (ELAN P075).

\section{References}

1. WHO. https://www.who.int/en/activities/tracking-SARS-CoV-2-variants. (2022).

2. Wang, L. \& Cheng, G. Sequence analysis of the emerging SARS-CoV-2 variant Omicron in South Africa. Journal of Medical Virology n/a.

3. Hoffmann, M. et al. SARS-CoV-2 Cell Entry Depends on ACE2 and TMPRSS2 and Is Blocked by a Clinically Proven Protease Inhibitor. Cell 181, 271-280 e8 (2020).

4. Yang, J. et al. Molecular interaction and inhibition of SARS-CoV-2 binding to the ACE2 receptor. Nat Commun 11, 4541 (2020).

5. $\quad \mathrm{Li}, \mathrm{W}$. et al. Angiotensin-converting enzyme 2 is a functional receptor for the SARS coronavirus. Nature 426, 450-4 (2003).

6. Jackson, C.B., Farzan, M., Chen, B. \& Choe, H. Mechanisms of SARS-CoV-2 entry into cells. Nat Rev Mol Cell Biol 23, 3-20 (2022).

7. Zhou, P. et al. A pneumonia outbreak associated with a new coronavirus of probable bat origin. Nature 579, 270-273 (2020).

8. Zhou, T. et al. Cryo-EM Structures of SARS-CoV-2 Spike without and with ACE2 Reveal a pHDependent Switch to Mediate Endosomal Positioning of Receptor-Binding Domains. Cell Host Microbe 28, 867-879 e5 (2020).

9. Socher, E. et al. Computational decomposition reveals reshaping of the SARS-CoV-2-ACE2 interface among viral variants expressing the N501Y mutation. J Cell Biochem 122, 1863-1872 (2021).

10. Socher, E. et al. Mutations in the B.1.1.7 SARS-CoV-2 Spike Protein Reduce Receptor-Binding Affinity and Induce a Flexible Link to the Fusion Peptide. Biomedicines $\mathbf{9 ( 2 0 2 1 ) .}$

11. Jawad, B., Adhikari, P., Podgornik, R. \& Ching, W.Y. Key Interacting Residues between RBD of SARS-CoV-2 and ACE2 Receptor: Combination of Molecular Dynamics Simulation and Density Functional Calculation. J Chem Inf Model 61, 4425-4441 (2021).

12. ECDC. https://www.ecdc.europa.eu/en/covid-19/variants-concern. (2021).

13. Khan, M.I. et al. Impact of the Double Mutants on Spike Protein of SARS-CoV-2 B.1.617 Lineage on the Human ACE2 Receptor Binding: A Structural Insight. Viruses 13(2021).

14. Deng, X. et al. Transmission, infectivity, and antibody neutralization of an emerging SARS-CoV2 variant in California carrying a L452R spike protein mutation. medRxiv (2021).

15. Wilhelm, A. et al. Antibody-Mediated Neutralization of Authentic SARS-CoV-2 B.1.617 Variants Harboring L452R and T478K/E484Q. Viruses 13(2021).

16. Focosi, D. \& Maggi, F. Neutralising antibody escape of SARS-CoV-2 spike protein: Risk assessment for antibody-based Covid-19 therapeutics and vaccines. Rev Med Virol 31, e2231 (2021).

17. Liu, C. et al. Reduced neutralization of SARS-CoV-2 B.1.617 by vaccine and convalescent serum. Cell 184, 4220-4236.e13 (2021).

18. Wilhelm, A. et al. Reduced Neutralization of SARS-CoV-2 Omicron Variant by Vaccine Sera and monoclonal antibodies. medRxiv, 2021.12.07.21267432 (2021).

19. Dejnirattisai, W. et al. Reduced neutralisation of SARS-CoV-2 omicron B.1.1.529 variant by post-immunisation serum. Lancet (2021).

20. Dejnirattisai, W. et al. The antigenic anatomy of SARS-CoV-2 receptor binding domain. Cell 184, 2183-2200 e22 (2021). 
21. ECDC. Implications of the emergence and spread of the SARS CoV 2 B.1.1. 529 variant of concern (Omicron) for the EU/EEA. (2021).

22. Starr, T.N. et al. Deep Mutational Scanning of SARS-CoV-2 Receptor Binding Domain Reveals Constraints on Folding and ACE2 Binding. Cell 182, 1295-1310 e20 (2020).

23. Zhu, X. et al. Cryo-electron microscopy structures of the N501Y SARS-CoV-2 spike protein in complex with ACE2 and 2 potent neutralizing antibodies. PLoS Biol 19, e3001237 (2021).

24. Wadowski, P.P. et al. Glycocalyx as Possible Limiting Factor in COVID-19. Front Immunol 12, 607306 (2021).

25. Lang, J. et al. Inhibition of SARS pseudovirus cell entry by lactoferrin binding to heparan sulfate proteoglycans. PLoS One 6, e23710 (2011).

26. Clausen, T.M. et al. SARS-CoV-2 Infection Depends on Cellular Heparan Sulfate and ACE2. Cell 183, 1043-1057 e15 (2020).

27. Guex, N. \& Peitsch, M.C. SWISS-MODEL and the Swiss-PdbViewer: an environment for comparative protein modeling. Electrophoresis 18, 2714-23 (1997).

28. Jurrus, E. et al. Improvements to the APBS biomolecular solvation software suite. Protein Science 27, 112-128 (2018).

29. Case, D.A. et al. AMBER 2020.

30. Maier, J.A. et al. ff14SB: Improving the Accuracy of Protein Side Chain and Backbone Parameters from ff99SB. J Chem Theory Comput 11, 3696-713 (2015).

31. Jorgensen, W.L., Chandrasekhar, J., Madura, J.D., Impey, R.W. \& Klein, M.L. Comparison of simple potential functions for simulating liquid water. The Journal of Chemical Physics 79, 926935 (1983).

32. Götz, A.W. et al. Routine Microsecond Molecular Dynamics Simulations with AMBER on GPUs. 1. Generalized Born. J Chem Theory Comput 8, 1542-1555 (2012).

33. Salomon-Ferrer, R., Götz, A.W., Poole, D., Le Grand, S. \& Walker, R.C. Routine Microsecond Molecular Dynamics Simulations with AMBER on GPUs. 2. Explicit Solvent Particle Mesh Ewald. J Chem Theory Comput 9, 3878-88 (2013).

34. Le Grand, S., Götz, A.W. \& Walker, R.C. SPFP: Speed without compromise-A mixed precision model for GPU accelerated molecular dynamics simulations. Computer Physics Communications 184, 374-380 (2013).

35. Berendsen, H.J.C., Postma, J.P.M., Gunsteren, W.F.v., DiNola, A. \& Haak, J.R. Molecular dynamics with coupling to an external bath. The Journal of Chemical Physics 81, 3684-3690 (1984).

36. Jean-Paul Ryckaert, G.C., Herman J.C Berendsen,. Numerical integration of the cartesian equations of motion of a system with constraints: molecular dynamics of $n$-alkanes. Journal of Computational Physics 23, 327-341 (1977).

37. Hopkins, C.W., Le Grand, S., Walker, R.C. \& Roitberg, A.E. Long-Time-Step Molecular Dynamics through Hydrogen Mass Repartitioning. J Chem Theory Comput 11, 1864-74 (2015).

38. Roe, D.R. \& Cheatham, T.E., 3rd. PTRAJ and CPPTRAJ: Software for Processing and Analysis of Molecular Dynamics Trajectory Data. J Chem Theory Comput 9, 3084-95 (2013).

39. Pettersen, E.F. et al. UCSF Chimera-A visualization system for exploratory research and analysis. Journal of Computational Chemistry 25, 1605-1612 (2004).

40. Barnes, C.O. et al. SARS-CoV-2 neutralizing antibody structures inform therapeutic strategies. Nature 588, 682-687 (2020).

41. Hansen, J. et al. Studies in humanized mice and convalescent humans yield a SARS-CoV-2 antibody cocktail. Science 369, 1010-1014 (2020). 
432 Figure S1:

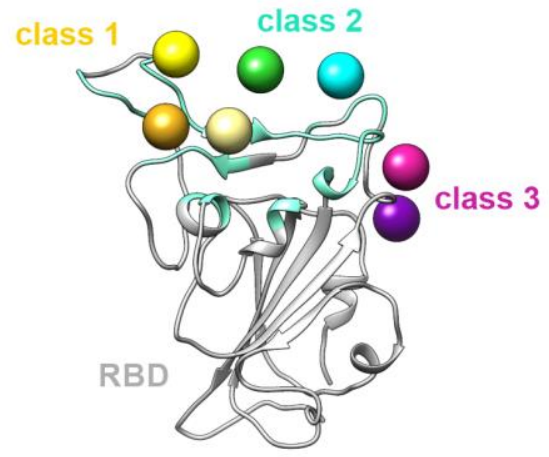

b

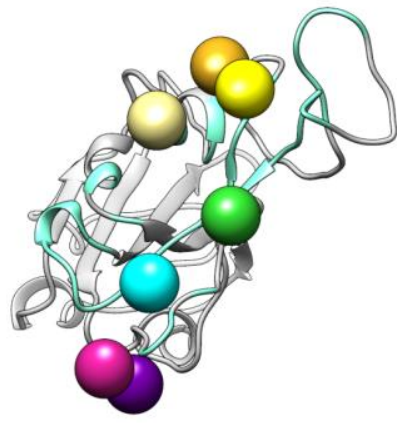

C
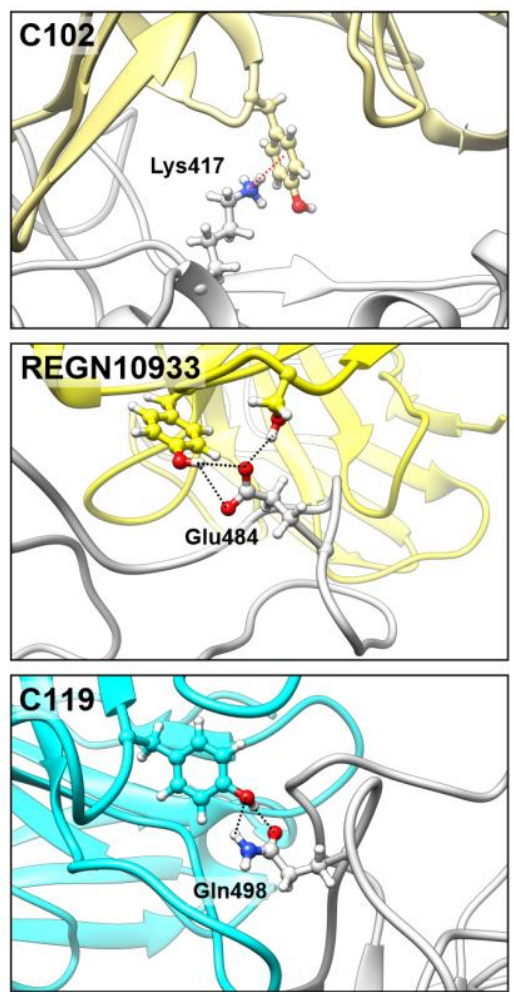
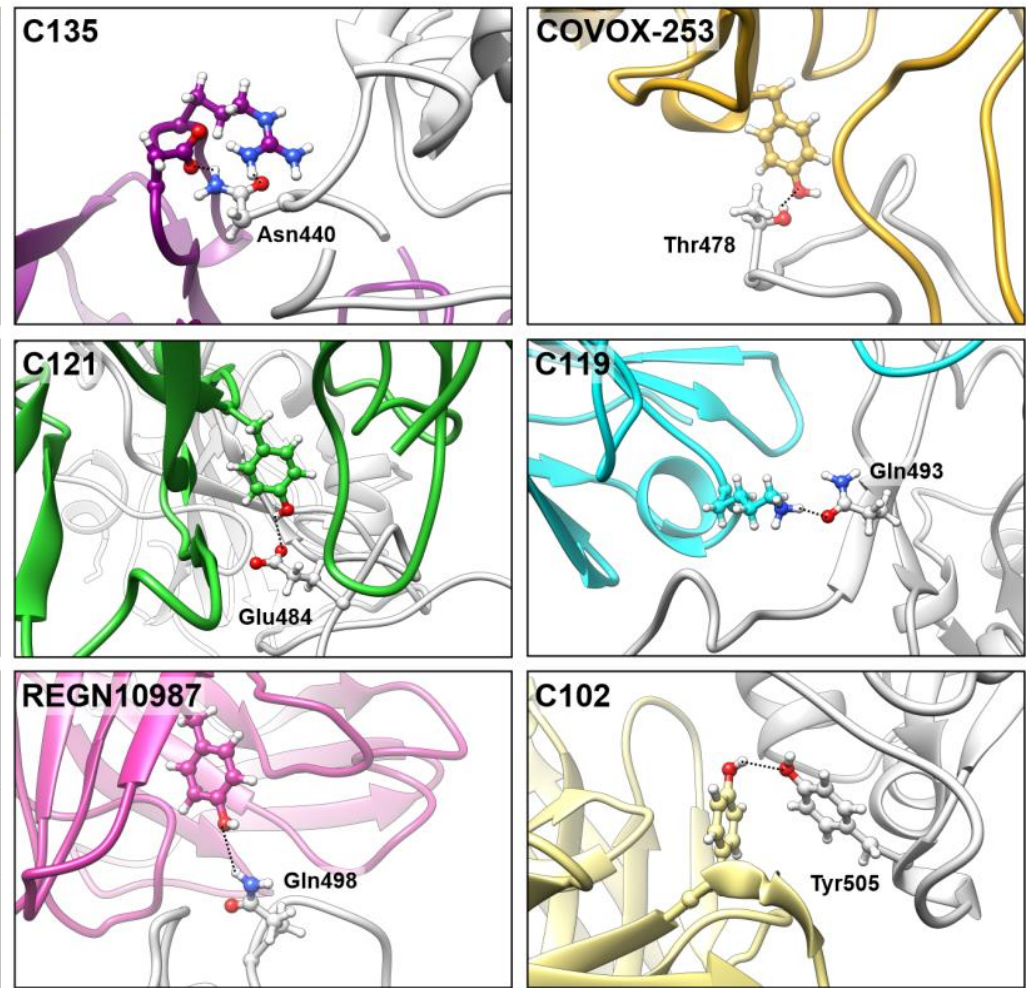

Figure S1: Neutralizing antibodies from class 1, class 2 and class $3^{40}$ interact with residues in wild type spike protein which are mutated in the RBD of the delta and/or the omicron variant. a, RBD shown in grey with residues forming the interaction interface with ACE2 highlighted in aquamarine. The colored spheres represent the center of the binding site of the seven exemplarily selected neutralizing antibodies. $\mathbf{b}$, As in figure panel $\mathbf{a}$ but structure was rotated to show from a different view. c, Detailed structural representation of RBD interface residues in wild type and the interacting residues of the neutralizing antibodies C102 (PDB ID code: 7K8M) ${ }^{40}$, C135 (PDB ID code:7K8Z) ${ }^{40}$, COVOX-253 (PDB ID code:7BEN) ${ }^{17}$, REGN10933 (PDB ID code: 6XDG) ${ }^{41}$, C121 (PDB ID code: 7K8Y) ${ }^{40}$, C119 (PDB ID code: 7K8W) ${ }^{40}$ and REGN10987 (PDB ID code: 6XDG) ${ }^{41}$ (colors were used as in a). Exemplary structural illustrations are shown only for RBD residues mutated in the delta and/or omicron variants. Dashed lines indicate intermolecular hydrogen bonds or as in the case of Lys417 intermolecular cation-pi interactions. 
449 Figure S2:

a contacts between individual RBD residues and ACE2

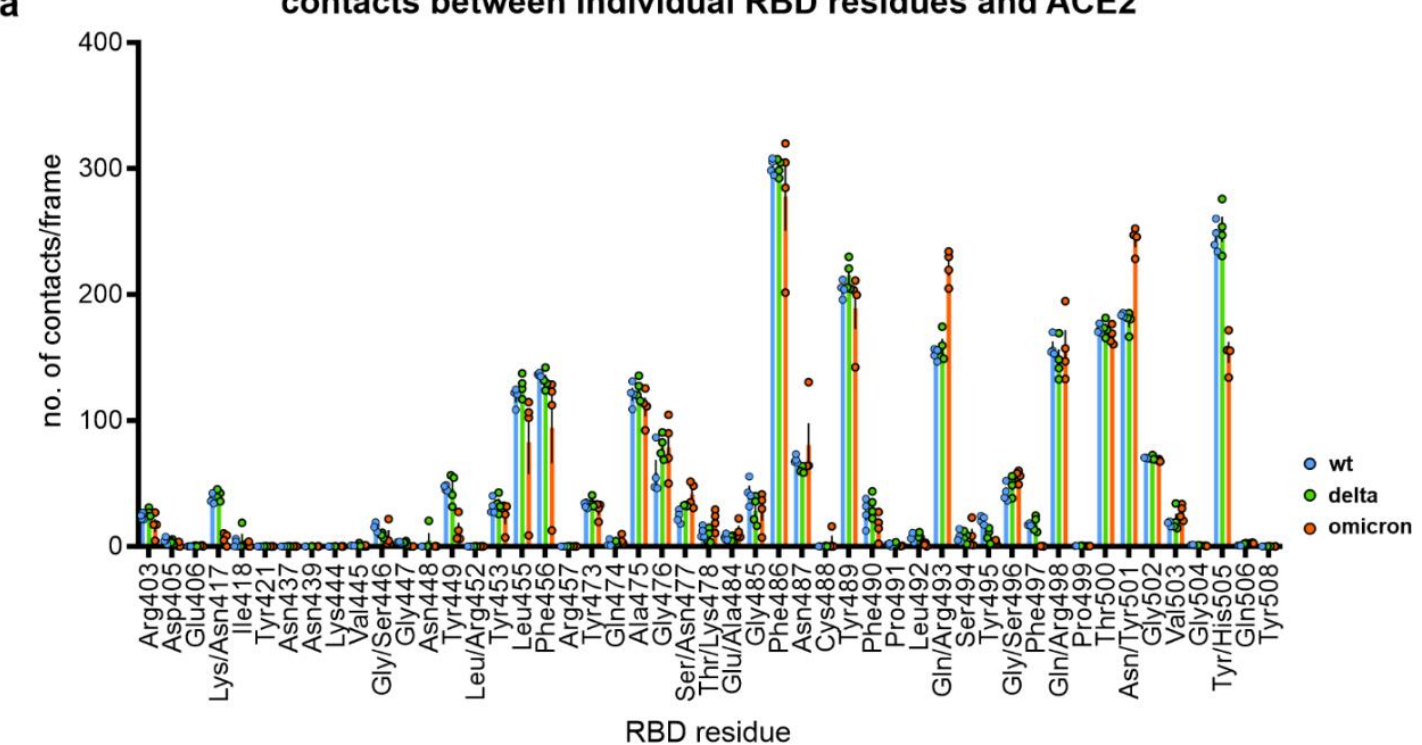

b

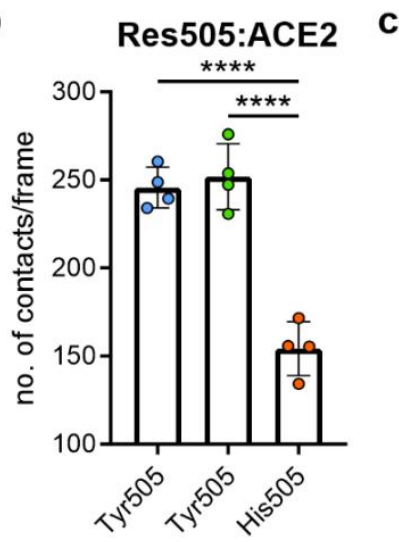

d

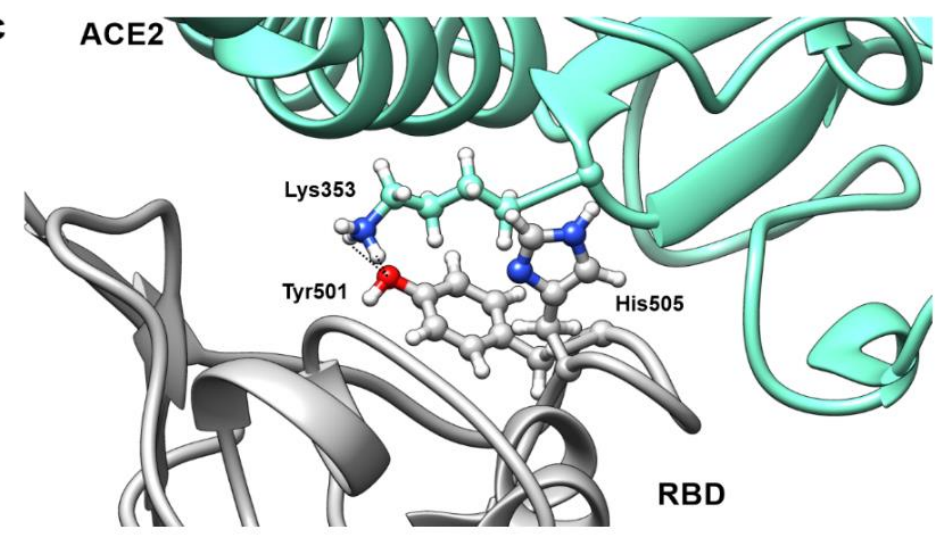

epsilon (B.1.427/B.1.429)

kappa (B.1.617.1)
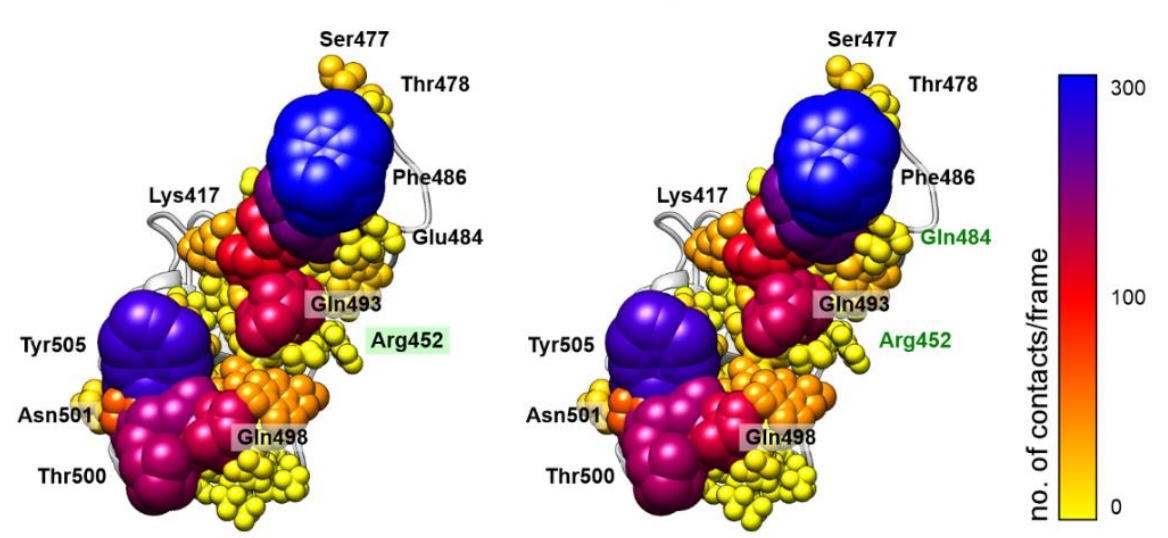

Figure S2: a, Number of contacts for all residues of the RBD within 8 Å distance to ACE2 for wt, delta and omicron variants. b, Number of contacts for residue 505 (tyrosine for wt and delta, histidine for omicron) formed with ACE2. c, Structural representation of tyrosine 501 (Tyr501) and histidine 505 (His505) expressed on the RBD or omicron and lysine 353 expressed on ACE2. d, Structural representation of number of contacts formed by the epsilon (B.1.427/B.1.429) variant and the kappa (B.1.617.1) variant. Residues are shown in different color and sphere size. 
457 Figure S3:

energy between individual RBD residues and ACE2

$\mathrm{RBD}$ residue

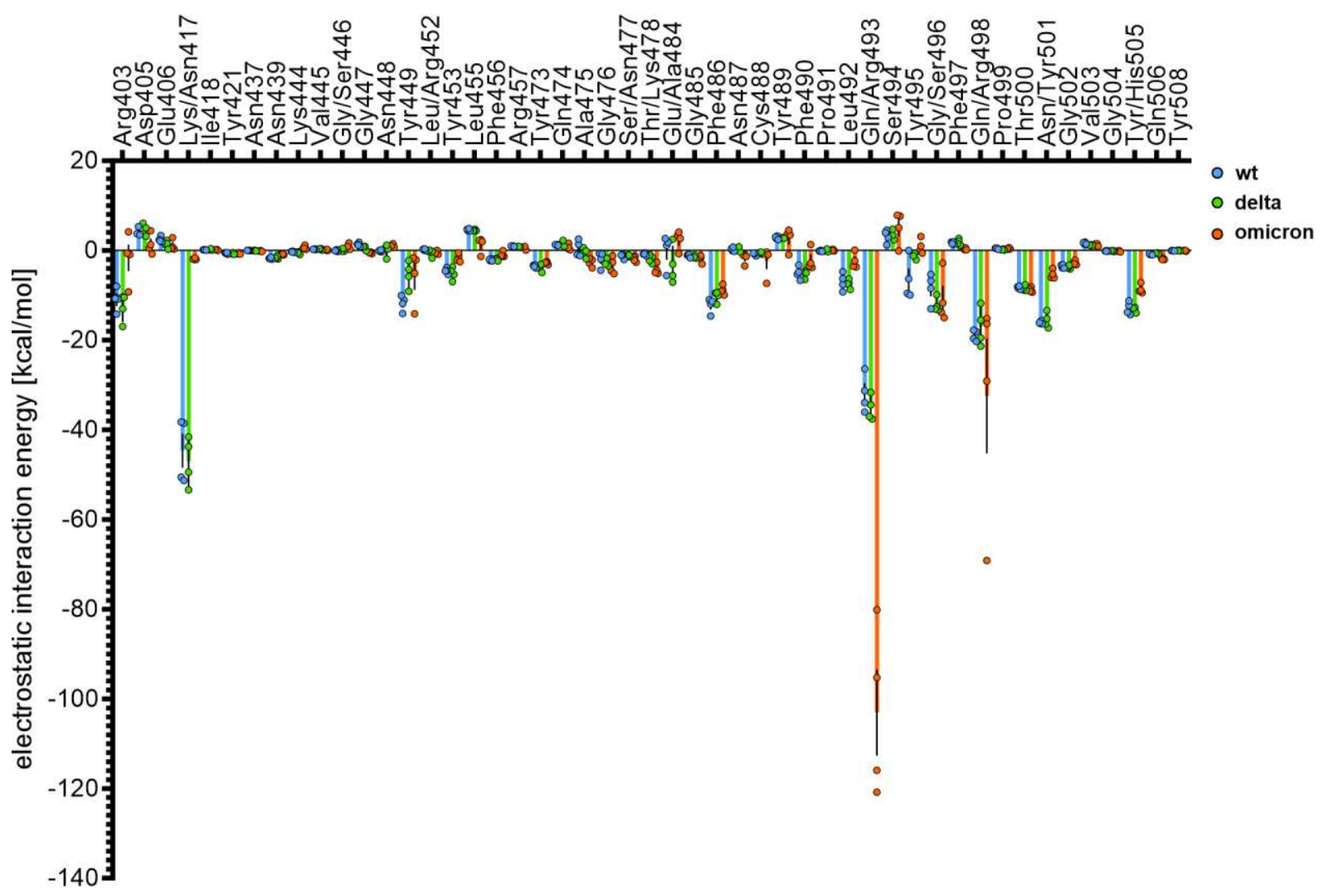

459 Figure S3: Linear electrostatic interaction energy for individual RBD residues expressed on the RBD-ACE2 inter460 face (within $8 \AA$ distance from ACE2).

461 
Figure S4:

a

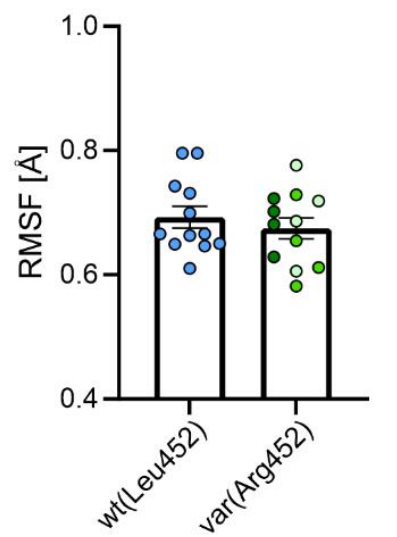

C

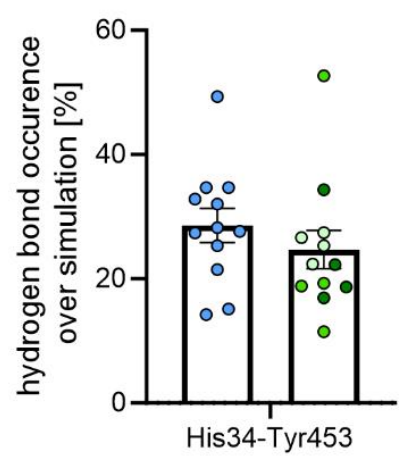

b GIn493 side chain atoms

\section{wt}

epsilon (B.1.427/B.1.429)

delta (B.1.617.2)

- kappa (B.1.617.1)

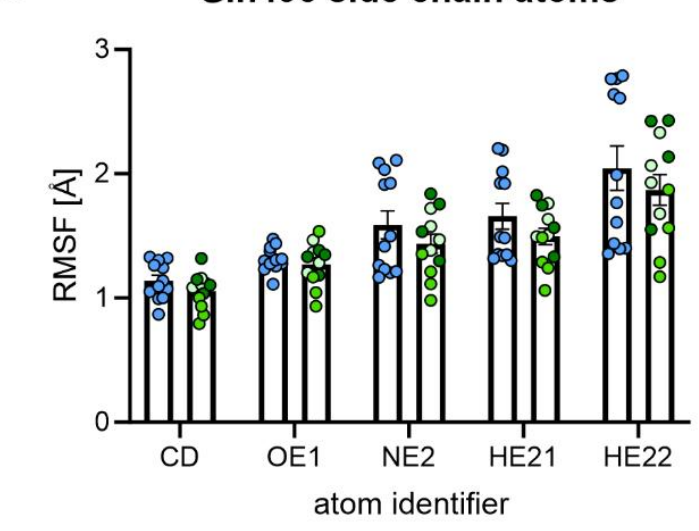

463

464

465

466

467

468

469

470

471

472

473

474

475

476

477

478

479

480 d

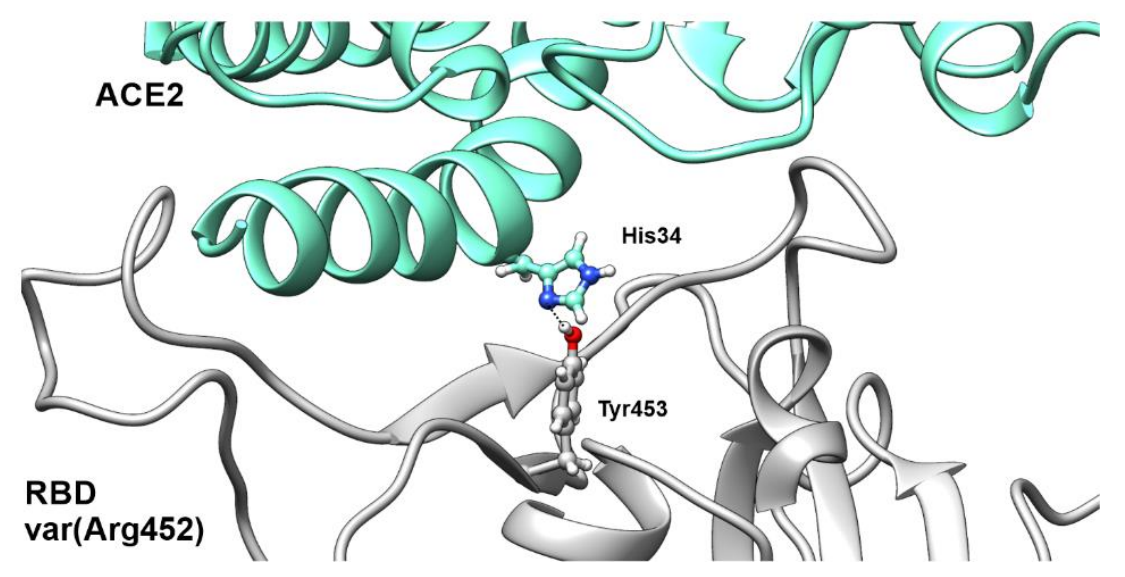

Figure S4: a, Pooled comparison of the RMSF (root mean square fluctuation) values for glutamine 493 comparing wt and L452R expressing variants. b, Detailed RMSF analysis of the side chain atoms of glutamine 493 comparing wt and L452R expressing variants (epsilon: light green, delta: medium green, kappa: dark green). c, Hydrogen bond occurrence between tyrosine 453 (Tyr453, RBD) and histidine 34 (His34, ACE2). d, Structural representation of the hydrogen bond formed between tyrosine 453 (Tyr453, RBD with L452R) and histidine 34 (His34, ACE2). 
Figure S5:

a
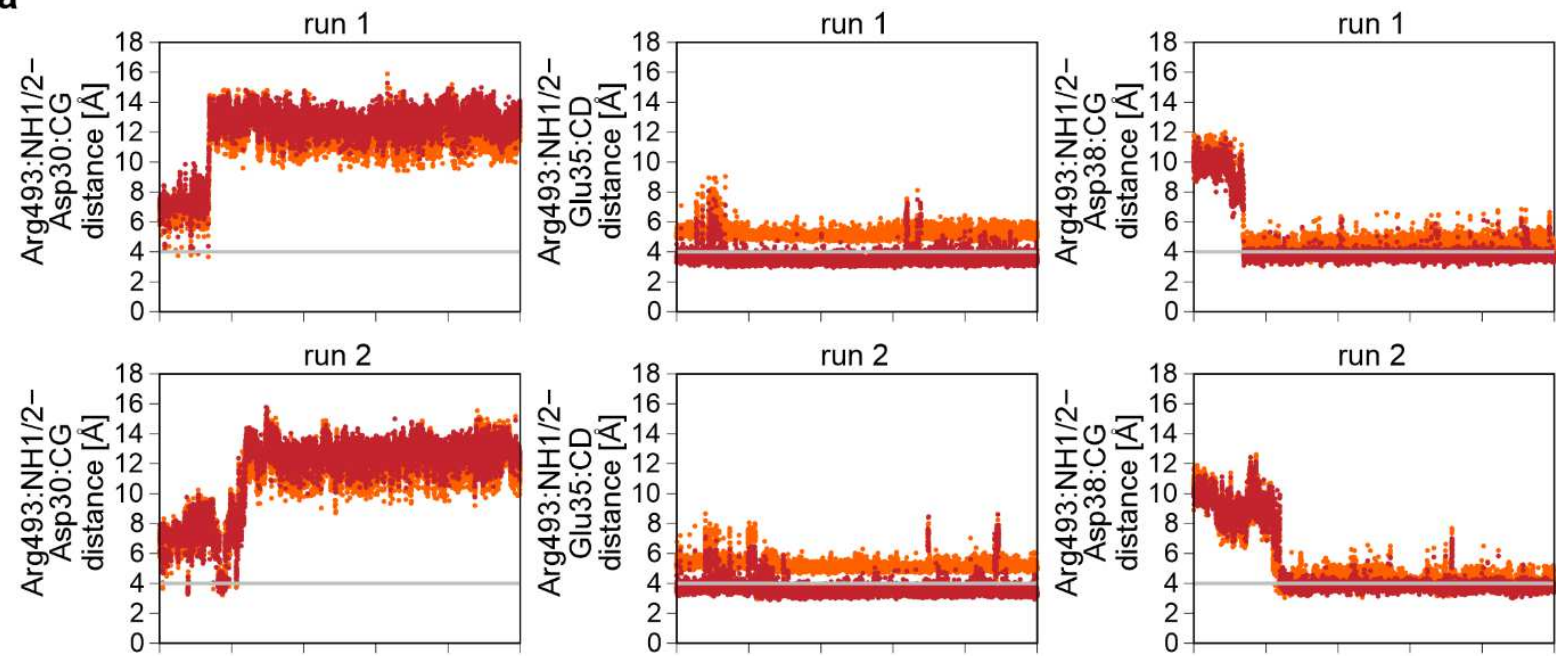

run 2
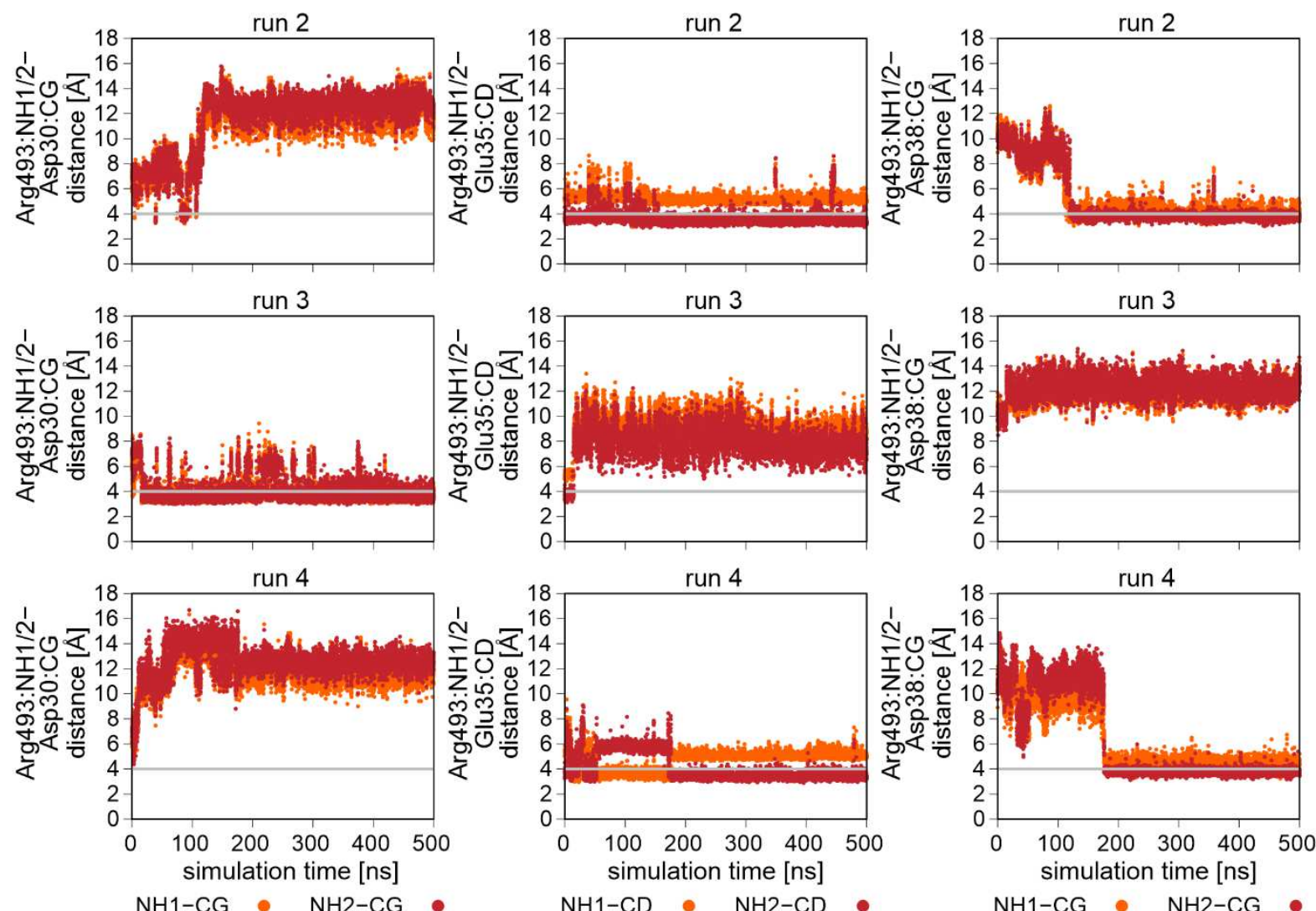

b

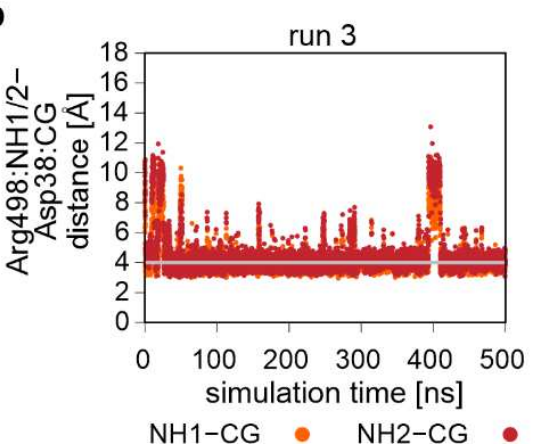

Figure S5: a, Time resolved distance plots of for individual MD simulation runs with distances plotted between arginine 493 (Arg493) expressed on the RBD and aspartate 30 (Asp30), glutamate 35 (Glu35) and aspartate 38 (Asp38, all expressed on ACE2). b, time resolved distance plot from run 3 (a) for arginine 498 (Arg498) expressed on the RBD and aspartate 38 (Asp38) expressed on ACE2. 
Figure S6:

a

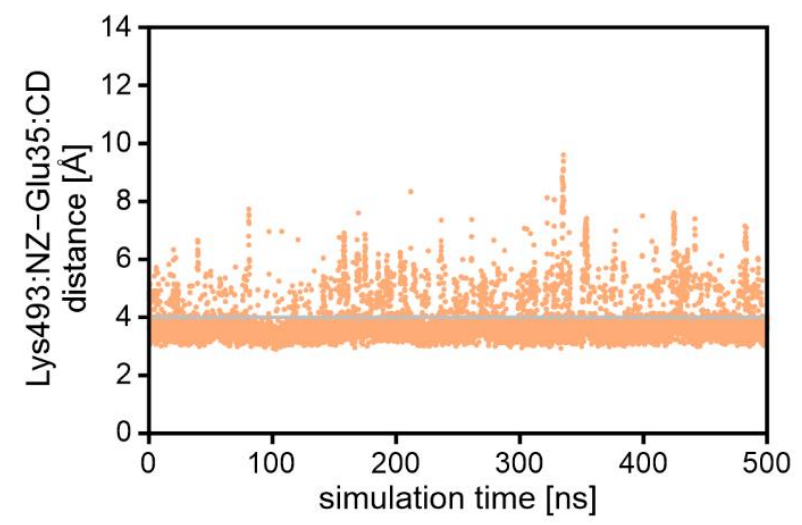

C

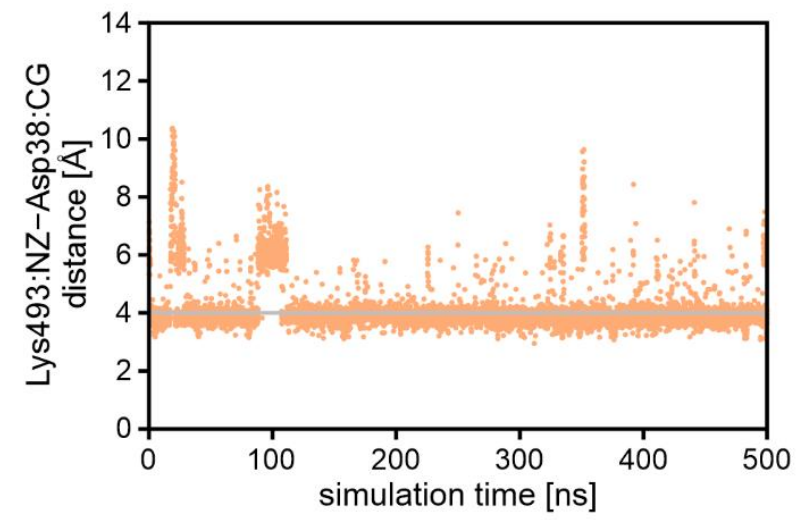

b

Lys493-Glu35

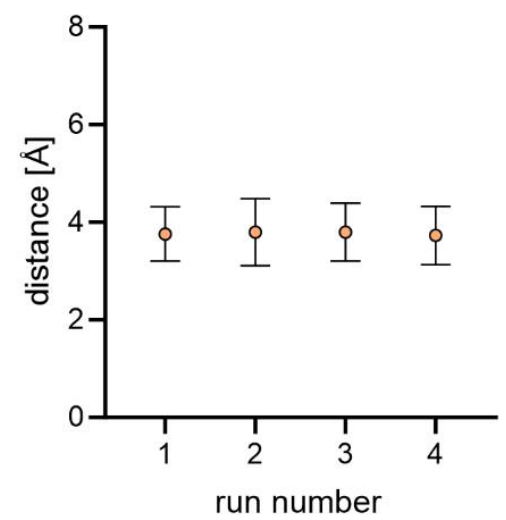

d

Lys493-Asp38

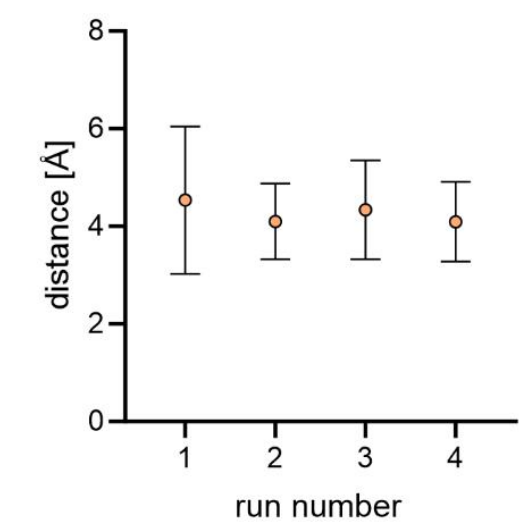

f

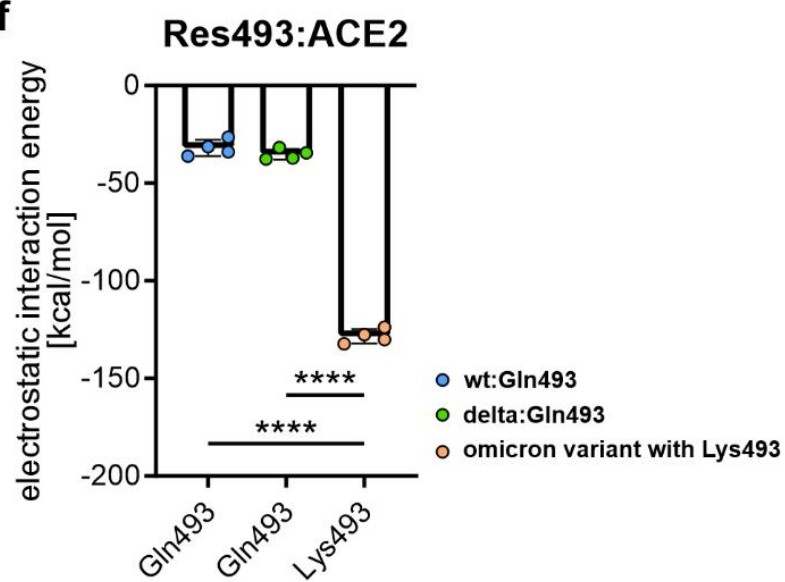

e

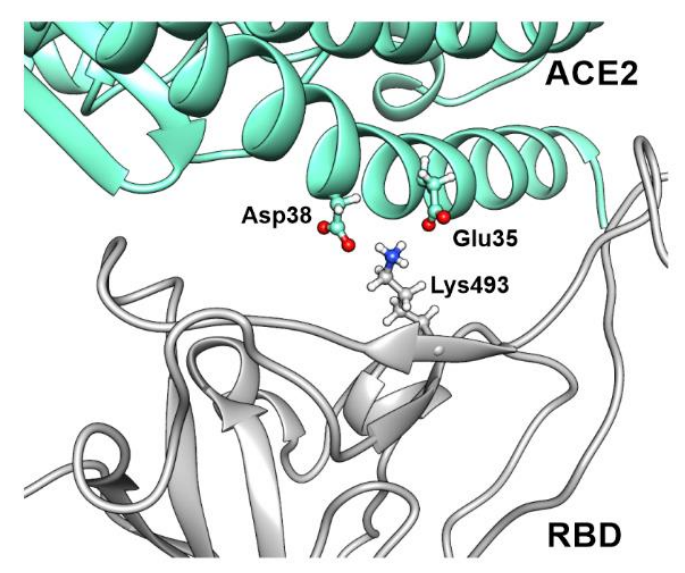

Figure S6: a, Representative distance plot of lysine 493 (RBD, omicron) and glutamate 35 (ACE2). b, Average distance with standard deviation error bars shown for four individual $500 \mathrm{~ns}$ runs for residues lysine 493 (RBD, omicron) and glutamate 35 (ACE2). c, Representative distance plot for lysine 493 (RBD, omicron) and aspartate 38 (ACE2). d, Average distance with standard deviation error bars shown for four individual 500 ns runs for residues lysine 493 (RBD, omicron) and aspartate 38 (ACE2). e, Structural representation of the two newly formed salt bridges between lysine 493 (Lys493) on the RBD and glutamate 35 (Glu35) and aspartate 38 (Asp38) both expressed on ACE2. f, Linear interaction energy for residue 493 analyzed for wt (blue), delta (green) and omicron (orange; ${ }^{* * * *} \mathrm{p}<0.0001$, one-way ANOVA). 